\title{
VIEWS OF CONSTRUCTION PROFESSIONALS' ON THE CAUSES AND REMEDIES OF BUILDING COLLAPSE IN NIGERIA
}

\author{
Nwabueze Michael Anosike $\mathrm{U}^{1}$

\footnotetext{
${ }^{1}$ Department of Building, School of Environmental Sciences, Federal University of Technology, Owerri, Nigeria
}
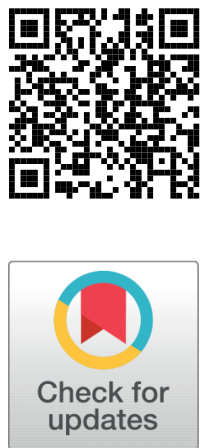

Received 11 June 2021

Accepted 22 June 2021

Published 30 June 2021

Corresponding Author

Nwabueze Michael Anosike, anosi

ken932@gmail.com

DOI $10.29121 /$

ijetmr.v8.i6.2021.976

Funding: This research received no specific grant from any funding agency in the public, commercial, or not-for-profit sectors.

Copyright: (C) 2021 The Author(s). This is an open access article distributed under the terms of the Creative Commons Attribution License, which permits unrestricted use, distribution, and reproduction in any medium, provided the original author and source are credited.

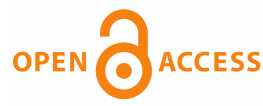

\section{ABSTRACT}

The collapse of building has been an issue of great concern to all and sundry in Nigeria and yet these buildings are major contributors to the development of any nation. Therefore, when a building collapses, it fails to render the functions which it should perform to the people and the government. A building is regarded as collapsed if the whole structure or part thereof has fallen down and therefore cannot serve the purpose for which it was intended. When building collapses, it generates deep emotional reactions from every segment of the population constituting great losses to the country in terms of physical, human and material resources. Therefore, the aim of this research is to assess the opinion of construction professionals who are perceived as critical stakeholders on the causes as well as to proffer remedies to curb the menace. The case study is Owerri municipal council, Imo State, Nigeria. A well-structured four-point Likert Scale questionnaire was drawn and administered to 250 selected sampled respondents to elicit data. Out of that the 250 respondents, 193 representing $77 \%$ valid respondents were retrieved and utilized in the study analysis. The analysis revealed that all critical construction stakeholders were implicated as contributors towards the incidences of building collapse. The study recommends among others that, Government, professionals' as well as other critical stakeholders should adhere strictly to their statutory roles in order to curb the menace of building collapse in Nigeria.

Keywords: Building Collapse, Construction Industry, Government, Professionals', Stakeholders

\section{INTRODUCTION}

The ever-mounting crisis in the housing sector of Nigeria has been in various dimensions, which range from absolute housing unit shortages, to the emergence and proliferation of the squatter settlements and slums, the rising cost of housing rent and the building collapse. The collapse of building has been an issue of great concern to all and sundry in Nigeria and yet these buildings are major contributors to the 
development of any nation. Therefore, when a building collapses, it fails to render the functions which it should perform to the people and the government. A building is regarded as collapsed if the whole structure or part thereof has fallen down and therefore cannot serve the purpose for which it was intended. When building collapses, it generates deep emotional reactions from every segment of the population constituting great losses to the country in terms of physical, human and material resources Ayedun et al. (2012); Nbrri (2020). The incidence of building collapse in Nigerian is reaching an epidemic proportion. These incidences have resulted in the loss of lives and property, which have also infringed permanent disabilities to many. Though building collapse is not peculiar to Nigeria, the trend in the country is becoming quite worrisome and a source of concern to stakeholders. According to Ayedun et al. (2012) the spate and frequency of occurrence have become a major source of concern not only to the governments but to all well-meaning Nigerians and most especially the stakeholders in the building industry as the magnitude of the incidents are becoming very unprecedented. That building collapse incidence is still regularly occurring despite the fact that there has been the increasing diffusion of engineering knowledge over the years have brought to question whether these stakeholders have critically examined the reasons for building failure and the roles they can play or the strategies they can articulate that would help to arrest the incidents.

\section{PROBLEM STATEMENT}

The increasing report of building collapse in Imo State particularly in Owerri municipal council has left so many questions unanswered as to what the government is doing to avert the menace. The unfortunate trending situation may be ascribed to negligence by governments at all levels, professional bodies of the built environment in Nigeria as well as the private sector and even the consumers of the final product of the industry, all of who are the critical stakeholders in the built environment sector of the economy. Based on the alarming situation of rampant collapse of buildings in Nigeria, this study intends to provide answers as to why is collapse not a major problem to Nigeria government; what have all the professional bodies associated to build environment done to mitigate building collapse and have there been penalty(ies) meted out to the indicted company, organization or client and contractors or even consultants or professionals who may have been indicted? The researcher believes that if these highlighted questions are adequately answered in the study, the solution towards curbing the menace of building collapse would be ameliorated if not eradicated. The main thrust of this study therefore is to compare the views of construction professionals within Owerri Municipal Council, Imo State on the causes and remedies of building collapse in Nigeria, and this will be achieved through the following objectives, to identify and statistically rank the causes of building collapse within Owerri municipal area, determine the possible ways of minimizing and/or militating collapse, suggest effective regulatory controls for building compliance to national 
building code to mitigate collapse.

\section{LITERATURE REVIEW}

\subsection{BUILDING COLLAPSE AS A WORLDWIDE PHENOMENON}

Building collapse is a worldwide phenomenon. Even among the advanced countries, there have been reported cases of collapsed buildings. Quite recently in Rio de Janeiro, Argentina, the Associated Press on 30th January, 2012 reported that fire fighters pulled out bodies from the rubbles of 3 collapsed buildings. And earlier in September 30, 2010, a 13-Storey building toppled over in China. However, the regularity and causes of occurrence differ from one clime to another. While the causes of building collapse in most other countries are traceable to natural forces (earthquakes, tsunamis, floods, etc), in Nigeria, its occurrence is mainly due to subjective factors (negligence, Incompetence, greed, etc) on the part of the professionals and promoters of such building works Okereke et al (2005) cited in Ezeanyika (2018). The regularity of collapsed buildings across the country is quite alarming and a source of great concern to the built environment professionals and the general public, because of the enormous loss of life and property, each time a building collapsed. Records show that between 1971 to the present, the average number of reported cases of building collapse is not less than 10 annually with not less than 15 lives lost and properties worth millions of Naira wasted.

\subsection{BUILDING COLLAPSE}

According to the dictionary of Architecture and Construction refers to collapse as mechanical failure. Collapse is a state of complete failure, when the structure has literally given way and most members have caved-in, crumbled or buckled; the building can no longer stand as originally built Dimuna (2010). A structure is a whole building, complex framework or essential part of a building. According to Fakere et al. (2012) structure is defined as a body capable of resisting applied loads without any deformation of part relative to one another. The structure of the building is therefore that part of the building construction which gives the construction sufficient strength to withstand the load to which the load is subjected. Building collapse can simply be defined as a total or a partial/progressive failure of one or more components of a building leading to the inability of the building to perform its principal function of comfort, satisfaction, safety and stability Olagunju et al. (2013). A building may collapse when one or more of its essential components fail Fakere et al. (2012). Building failure is defined as an act of omission or occurrence or performance. Failure could also be defined as non-occurrence, non-performance, running short, breaking down, ill-success, insolvency and unsuccessful attempt Ayuba et al. (2012). Building failure could be of two types namely; cosmetic failure that occurs when something has been added to or subtracted from the building, thus affecting the structures outlooks while structural failures affect both the outlook and structural stability of the build- 
ing Ayuba et al. (2012). Hence, failure occurs when a building is not able to perform the function Ukpata and Sunday 15th October (2006).

\subsection{CAUSES OF BUILDING COLLAPSE}

Ayininuola and Olalusi (2005) opines that in Nigeria, building failure have been attributed to causes such as design faults (50\%), faults on construction site (40\%) and product failure (10\%). Hall (1984) on the other hand attributed faulty design, faulty execution of work and use of faulty materials to be major causes of building collapse. Some schools of thought classify the causes as physical factors, ecological status of the site, composition of technical components, social factors, economic factors, engineering factors, human factors, government policies, and political factors. underpins environmental changes, natural and manmade hazards, improper presentation and interpretation in the design as causes of building collapse. In the same light Ukpata and Sunday 15th October (2006) identifies causes of building failures to poor structural design by quacks, lack of structural design, use of substandard materials, negligence of the use of appropriate personnel in building construction supervision, poor workmanship and supervision. A very Senior News Correspondent with the Consumer News media in 2012 during an interview highlighted other common causes of building collapse are bad design, faulty construction, foundation failures, extra-ordinary loads, use of unqualified contractors and poor monitoring and above all, lack of enforcement of building codes by the relevant government officials.

Apart from the above known causes, the Nigerian factor becomes a prominent issue to contend with. The Nigerian factor in the building industry comes in various dimensions such as corruption, lawlessness and our presumptions that any engineer or professionals in the built environment can assume all forms of responsibility in a building process without the basic skills required for it. Corruption is made manifest in greed and tendency to cheat in virtually every aspect, starting from poor materials and quality of work to the quantities we adopt. The use of unskilled labour, inexperienced professionals, tendency of some professionals to cross-carpet to lucrative specialists' duties where they lack skills, ignorance and the proliferation of quacks in the building industry are all facts to contend with Ede (2000). Global Corruption Report (2005) cited in Ezeanyika (2018), Anosike and Inyang-Udoh (2015) identified corruption as one factor that has caused high cost of building materials and reduction in standards of construction works in the developing economy. The Global Corruption report observes that corruption may be at different stages namely, at contract award, planning \& design, construction stage s respectively, and when the building is completed. It may take different forms like bribery Anosike and Inyang-Udoh (2015) deception and collusion Enenmoh and Anosike (2017) the end products of which are lowering of construction standard, increasing cost of repair and maintenance, defects on building that may not be discovered until its eventual collapse. Many buildings in Nigeria collapsed due to some of the following reasons; inadequate preliminary works, adoption of wrong foundation; poor concrete mix ratio; improper walling; 
lack of approved structural design; poor building material specification, ineffective supervision Fakere et al. (2012).

Dimuna (2010) casts a slur on the competence of the nation's building community of architects, structural engineers and builders - who are the professionals responsible for designing sites. These professionals are being attacked from all angles because of the recurring incidents of building collapse. But the building professionals should not bear the blame alone. This is because, firstly, it has been proved that owners of building under construction derail from their approved plans relying more on imagination and fantasy. Secondly, the approving authorities are also known to fail to monitor compliance with approved plans. Thirdly, some building owners shun professionals in order to cut costs. Fourthly, the high cost of building materials has led greedy contractors with eyes on profits, to patronize substandard materials. These short-cut measures have contributed immensely to the occurrence of failed buildings in the country. Deficient structural drawing, alteration of approved drawings, absence of proper supervision, building without approved building drawings, approval of technically deficient drawings, illegal alteration to existing buildings, absence of town planning inspection or monitoring of sites, clients penchants to cut corners, use of substandard materials, inefficient workmanship (labour), use of acidic and salty water, the activities of quacks, and clients' over reliance on contractors for making on sites.

Ayedun et al. (2012) attributed building failure to either natural or materials phenomena. A natural phenomenon may be attributable to earth quakes, storm/wind and typhoons while man-made phenomenon consists of disaster which may be borne out of man's negligence in areas such as soil type, building design and planning for extra loads and stress from winds and earthquake for tall buildings, foundation works, quality of building materials, lack or inadequate monitoring of craftsmen and poor quality of workmanship. Fredrick and James (1989) suggest that the overturning of structures due to heavy wind loads, sliding of structures due to high wind, roof uplift or sliding, and building sway due to lateral loads are major factors of failures of buildings. According to Ede (2000), activities necessary for the realization of efficient structures take place in the following fundamental stages: conceptual and design stage, construction-supervision stage and post construction service stage. A structure must be economical, safe, serviceable and aesthetically adequate Dimuna (2010). Any building, whether temporary, permanent or monumental structure must be properly planned, designed, constructed and maintained to realize the desired satisfaction, comfort, safety.

\subsection{REMEDIES TO CAUSES OF BUILDING COLLAPSE}

Based on the causes of building collapse identified in this study, it may be argued (and rightly) that the remedies are direct opposite of the causes. In the light of this some of the remedies to the causes of building collapse already identified are hereby highlighted as, discouraging the use of quacks for construction works, encourag- 
ing the use of reputable contractors for the building works, establishing "Developers' Aid Unit" in Government professional offices at all levels, prompt payment of certified Jobs by government and individuals, planned timely maintenance of property, compulsory implementation of National Housing Policy, discouraging property alterations and adherence to property use without obtaining appropriate approvals, encouraging soil geotechnical investigation, establishment of material testing laboratories across the country for easy reach, ensuring the compulsory use of professionals for project execution, encouraging the publication of results of enquiries on collapsed buildings, ensuring that adequate penalties are meted out to defaulting professionals and or professional bodies. In awarding contracts, governments should consider the employer-employee relationship and the law which assumes that anyone employed to execute construction work is trained and skilful enough to execute the project. It then behooves on anyone employing a contractor to ensure that the contractor has the expertise to carry out the work. Such knowledge is not the privilege of quacks. Quacks must not under any pretext be employed by any government or individual to undertake construction works. Prompt payment by the government at the local, state or federal level for certified jobs are important to enhance contractor's cash flow which will further encourage speedy and timely completion and delivery of projects. Governments must evolve an effective maintenance culture in order to prolong the values and life span of these construction products.

\section{Reported Cases of Building Collapse}

The review of reported cases is based on documented building collapses in Nigeria from 1974 to date by Chinwokwu (2000), Windapo (2006), and Dada (2006) cited in Ayedun et al. (2012). The listed incidents in the table below are not exhaustive but only show the extent and threat of building collapse in different zones and locations in Nigeria. The trend affected residential, religious and institutional buildings.

\begin{tabular}{|c|c|c|c|c|c|}
\hline $\mathbf{S} / \mathbf{N}$ & $\begin{array}{l}\text { Building } \\
\text { location }\end{array}$ & Type & Date & $\begin{array}{c}\text { Suspected } \\
\text { cause(s) }\end{array}$ & Life lost \\
\hline 1. & Mokola, Ibadam & $\begin{array}{c}\text { Multi-Storey } \\
\text { Building } \\
\text { Under } \\
\text { Construction }\end{array}$ & Oct. 1974 & $\begin{array}{l}\text { Excessive } \\
\text { Loading }\end{array}$ & 27 \\
\hline 2. & $\begin{array}{c}\text { Barnawa } \\
\text { Housing Estate } \\
\text { Kadurn }\end{array}$ & $\begin{array}{c}\text { Residential } \\
\text { Building }\end{array}$ & Aug. 1977 & Faulty Design & 28 \\
\hline 3. & $\begin{array}{c}\text { Barnawa } \\
\text { Housing Estate } \\
\text { Kaduna }\end{array}$ & $\begin{array}{c}3 \text { Storey } \\
\text { Residential } \\
\text { Building }\end{array}$ & 1977 & $\begin{array}{c}\text { Faulty Design } \\
\text { Carelessness }\end{array}$ & 6 \\
\hline 4. & $\begin{array}{c}\text { Govt. Sec. } \\
\text { School Markafu, } \\
\text { Kaduna }\end{array}$ & $\begin{array}{c}\text { School } \\
\text { Building }\end{array}$ & July 77 & $\begin{array}{c}\text { Faulty Design } \\
\text { Carelessness }\end{array}$ & 7 \\
\hline
\end{tabular}


Table 1 continued

\begin{tabular}{|c|c|c|c|c|c|}
\hline 5. & Iponri Lagos & $\begin{array}{l}\text { Uncompleted } \\
\text { 4- Storey } \\
\text { Building }\end{array}$ & $\begin{array}{c}18 \text { May } \\
1985\end{array}$ & $\begin{array}{c}\text { Excessive } \\
\text { Loading } \\
\text { /Carelessness }\end{array}$ & 13 \\
\hline 6. & $\begin{array}{c}\text { Ojelegba Rd. } \\
\text { Lagos }\end{array}$ & $\begin{array}{c}\text { Residential } \\
\text { Building }\end{array}$ & $\begin{array}{c}20 \text { May } \\
1985\end{array}$ & $\begin{array}{l}\text { Rainstorm } \\
\text { (Nature) }\end{array}$ & No Death \\
\hline 7. & Lagos Island & $\begin{array}{l}\text { Uncompleted } \\
\text { 3- Storey } \\
\text { Building }\end{array}$ & $\begin{array}{c}\text { 18th July } \\
1985\end{array}$ & $\begin{array}{c}\text { Excessive } \\
\text { Loading/ } \\
\text { Carelessness }\end{array}$ & $\begin{array}{l}9 \text { (All of } \\
\text { the same } \\
\text { family) }\end{array}$ \\
\hline 8. & Gboko, Benue & $\begin{array}{c}\text { Residential } \\
\text { Building }\end{array}$ & 1985 & $\begin{array}{c}\text { Carelessness of } \\
\text { The Owner) }\end{array}$ & $\begin{array}{c}2 \\
\text { Including }\end{array}$ \\
\hline 9. & $\begin{array}{l}\text { Adeniji Adele, } \\
\text { Lagos State }\end{array}$ & $\begin{array}{l}\text { Residential } \\
\text { Building }\end{array}$ & 1985 & $\begin{array}{l}\text { Carelessness of } \\
\text { The Owner }\end{array}$ & $\begin{array}{c}2 \\
\text { Including } \\
\text { Owner }\end{array}$ \\
\hline 10. & $\begin{array}{l}\text { Oshogbo, Osun } \\
\text { State }\end{array}$ & $\begin{array}{l}\text { Religious } \\
\text { (Mosques) } \\
\text { Building }\end{array}$ & May 1986 & $\begin{array}{c}\text { Faulty Design/ } \\
\text { Carelessness }\end{array}$ & 2 \\
\hline 11. & $\begin{array}{l}\text { Ona St. Enugu } \\
\text { State }\end{array}$ & $\begin{array}{l}\text { 2-Storey } \\
\text { Residential } \\
\text { Building }\end{array}$ & 1986 & $\begin{array}{c}\text { No } \\
\text { Investigation }\end{array}$ & 2 \\
\hline 12. & $\begin{array}{l}\text { Agege Lagos } \\
\text { State }\end{array}$ & $\begin{array}{c}\text { 2-Storey } \\
\text { Building } \\
\text { Under } \\
\text { Construction }\end{array}$ & $\begin{array}{c}\text { 9th May } \\
1987\end{array}$ & Carelessness & $\begin{array}{c}2 \\
\text { Including } \\
\text { Owner }\end{array}$ \\
\hline 13. & $\begin{array}{l}\text { Idumagbo Lane, } \\
\text { Idumota, Lagos }\end{array}$ & $\begin{array}{c}\text { 2-Storey } \\
\text { Residential } \\
\text { Building }\end{array}$ & $\begin{array}{c}\text { 14th May } \\
1987\end{array}$ & $\begin{array}{c}\text { Ignorant Client } \\
\text { (No Structural } \\
\text { Design }\end{array}$ & 17 \\
\hline 14. & $\begin{array}{c}\text { Ibadan Oyo } \\
\text { State }\end{array}$ & $\begin{array}{l}\text { 2-Storey } \\
\text { Building, } \\
\text { Public } \\
\text { Building - } \\
\text { Secondary } \\
\text { School }\end{array}$ & 1988 & Faulty Design & $\begin{array}{l}\text { Not } \\
\text { reported }\end{array}$ \\
\hline 15. & $\begin{array}{l}\text { Ikorodu Rd. } \\
\text { Lagos }\end{array}$ & $\begin{array}{l}\text { Commercial } \\
\text { Building }\end{array}$ & $\begin{array}{c}\text { 9th Oct. } \\
1987\end{array}$ & Storm (Nature) & 4 \\
\hline 16. & $\begin{array}{l}\text { Calabar, Cross } \\
\text { River State }\end{array}$ & $\begin{array}{c}\text { Residential } \\
\text { Building }\end{array}$ & Oct. 1989 & Storm (Nature) & 3 \\
\hline 17. & $\begin{array}{l}\text { Akinwunmi } \\
\text { Street, Mende, } \\
\text { Lagos State }\end{array}$ & $\begin{array}{l}\text { 6-Storey Hotel } \\
\text { Building }\end{array}$ & $\begin{array}{l}\text { 15th June } \\
1990\end{array}$ & $\begin{array}{c}\text { Faulty Design, } \\
\text { Carelessness }\end{array}$ & No Death \\
\hline 18. & $\begin{array}{l}\text { Iloabuchi, PH, } \\
\text { Rivers State }\end{array}$ & $\begin{array}{l}\text { A Primary } \\
\text { School } \\
\text { Building }\end{array}$ & July 1991 & $\begin{array}{c}\text { Bad } \\
\text { Construction }\end{array}$ & $\begin{array}{l}\text { Unreported } \\
\text { number }\end{array}$ \\
\hline 19. & $\begin{array}{l}\text { Port Harcourt, } \\
\text { Rivers State }\end{array}$ & $\begin{array}{l}\text { School } \\
\text { Building }\end{array}$ & $\begin{array}{c}1^{\text {st }} \text { Nov. } \\
1995\end{array}$ & $\begin{array}{c}\text { Ignorant } \\
\text { Owner (No } \\
\text { Structural } \\
\text { Design) }\end{array}$ & 55 \\
\hline 20. & $\begin{array}{c}\text { Agege, Lagos } \\
\text { State }\end{array}$ & $\begin{array}{l}\text { Church } \\
\text { Building }\end{array}$ & Jun 151990 & Faulty Design & 15 \\
\hline
\end{tabular}

Continued on next page 


\begin{tabular}{|c|c|c|c|c|c|}
\hline \multicolumn{6}{|c|}{ Table 1 continued } \\
\hline 21. & $\begin{array}{l}\text { Adeola Odeku } \\
\text { St. Victoria } \\
\text { Island Lagos }\end{array}$ & $\begin{array}{l}\text { 1-Storey } \\
\text { Building }\end{array}$ & 2000 & Storm (Nature) & No Death \\
\hline 22. & $\begin{array}{l}\text { Ojuelegba, } \\
\text { Lagos State }\end{array}$ & $\begin{array}{l}\text { 3-Storey } \\
\text { Building }\end{array}$ & April 2000 & Carelessness & 4 \\
\hline 23. & $\begin{array}{c}\text { Idi Oro Mushin } \\
\text { Lagos. }\end{array}$ & $\begin{array}{l}\text { Residential } \\
\text { Building }\end{array}$ & July 1989 & $\begin{array}{l}\text { Faulty Design, } \\
\text { Carelessness }\end{array}$ & 3 \\
\hline 24. & $\begin{array}{c}\text { Benin City, Edo } \\
\text { State }\end{array}$ & $\begin{array}{l}\text { 1-Storey } \\
\text { Building }\end{array}$ & Oct.1990 & $\begin{array}{c}\text { No } \\
\text { Investigation }\end{array}$ & No Death \\
\hline 25. & Abuja, FCT & $\begin{array}{l}\text { Multi-Storey } \\
\text { Building }\end{array}$ & March, 1993 & $\begin{array}{c}\text { Poor } \\
\text { supervision }\end{array}$ & $\begin{array}{l}\text { Unreported } \\
\text { number }\end{array}$ \\
\hline 26. & $\begin{array}{l}\text { Igbobi, Lagos } \\
\text { State }\end{array}$ & $\begin{array}{c}\text { Uncompleted } \\
\text { 3-Storey } \\
\text { Building }\end{array}$ & May 1995 & $\begin{array}{c}\text { No } \\
\text { Investigation }\end{array}$ & No Death \\
\hline 27. & $\begin{array}{c}\text { Alagbado, Ogun } \\
\text { State }\end{array}$ & $\begin{array}{l}\text { School } \\
\text { Building }\end{array}$ & May 1995 & $\begin{array}{c}\text { No } \\
\text { Investigation }\end{array}$ & No Death \\
\hline 28. & $\begin{array}{l}\text { Oke Igbala, } \\
\text { Lagos State }\end{array}$ & $\begin{array}{l}\text { 3-Storey } \\
\text { Church } \\
\text { Building }\end{array}$ & Oct. 1995 & $\begin{array}{c}\text { Faulty Design, } \\
\text { Carelessness }\end{array}$ & 15 \\
\hline 29. & $\begin{array}{l}\text { Oloowokere, } \\
\text { Oshodi, Lagos } \\
\text { State }\end{array}$ & $\begin{array}{l}\text { Apostolic } \\
\text { Church } \\
\text { Building }\end{array}$ & May 1996 & $\begin{array}{l}\text { Faulty Design, } \\
\text { Carelessness }\end{array}$ & 7 \\
\hline 30. & $\begin{array}{c}\text { Ijegbemi Street } \\
\text { Pedro, Lagos } \\
\text { State }\end{array}$ & $\begin{array}{c}\text { Classroom } \\
\text { Blocks Under } \\
\text { Construction }\end{array}$ & Oct. 1996 & $\begin{array}{c}\text { No } \\
\text { Investigation }\end{array}$ & 1 \\
\hline 31. & $\begin{array}{c}\text { Amu Street } \\
\text { Mushin, Lagos } \\
\text { State }\end{array}$ & $\begin{array}{l}\text { 2-Storey } \\
\text { Commercial } \\
\text { Building }\end{array}$ & June 1997 & $\begin{array}{c}\text { No } \\
\text { Investigation }\end{array}$ & None \\
\hline 32. & $\begin{array}{c}\text { Charity Rd, } \\
\text { Oko-Oba, Lagos } \\
\text { State }\end{array}$ & $\begin{array}{l}\text { 3-Storey } \\
\text { Building }\end{array}$ & June 1999 & $\begin{array}{c}\text { No } \\
\text { Investigation }\end{array}$ & None \\
\hline 33. & $\begin{array}{l}\text { Ojuelegba, } \\
\text { Lagos State }\end{array}$ & $\begin{array}{l}\text { Multi-Storey } \\
\text { building }\end{array}$ & 1999 & $\begin{array}{l}\text { Structural } \\
\text { failure }\end{array}$ & $\begin{array}{l}\text { Several } \\
\text { people }\end{array}$ \\
\hline 34. & $\begin{array}{c}\text { Four Square } \\
\text { Church, Abuja } \\
\text { FCT. }\end{array}$ & $\begin{array}{l}\text { 3-Storey } \\
\text { Building }\end{array}$ & Oct. 1999 & $\begin{array}{c}\text { No } \\
\text { Investigation }\end{array}$ & $\begin{array}{c}\text { Not } \\
\text { Available }\end{array}$ \\
\hline 35. & $\begin{array}{c}\text { Obawole Estate, } \\
\text { Lagos State }\end{array}$ & $\begin{array}{c}\text { 1-Storey } \\
\text { Building } \\
\text { Under } \\
\text { Construction }\end{array}$ & Oct. 1999 & $\begin{array}{c}\text { No } \\
\text { Investigation }\end{array}$ & None \\
\hline 36. & $\begin{array}{l}\text { Iju-Ishaga, } \\
\text { Lagos State }\end{array}$ & $\begin{array}{c}\text { 3-Storey } \\
\text { Building } \\
\text { Under } \\
\text { Construction }\end{array}$ & Oct. 1999 & $\begin{array}{c}\text { No } \\
\text { Investigation }\end{array}$ & 19 \\
\hline 37. & $\begin{array}{l}\text { Adeola Odeku } \\
\text { Street, Victoria } \\
\text { Island, Lagos }\end{array}$ & $\begin{array}{l}\text { One-Storey } \\
\text { Building }\end{array}$ & Oct. 1999 & Storm (Nature) & None \\
\hline 38. & $\begin{array}{l}\text { Idi-Oro Mushin, } \\
\text { Lagos State }\end{array}$ & $\begin{array}{l}\text { Residential } \\
\text { Building }\end{array}$ & 2000 & $\begin{array}{l}\text { Faulty Design } \\
\text { /Carelessness }\end{array}$ & $\begin{array}{c}\text { Not } \\
\text { Available }\end{array}$ \\
\hline
\end{tabular}


Table 1 continued

\begin{tabular}{|c|c|c|c|c|c|}
\hline 39. & $\begin{array}{c}\text { Eleganza Estate, } \\
\text { Ajah, Lagos } \\
\text { State }\end{array}$ & $\begin{array}{c}\text { Three-Storey } \\
\text { Building }\end{array}$ & April 2000 & $\begin{array}{l}\text { Incompetent } \\
\text { Contractor }\end{array}$ & 5 \\
\hline 40. & $\begin{array}{l}\text { P/Harcourt, } \\
\text { Rivers State }\end{array}$ & $\begin{array}{l}\text { Two-Storey } \\
\text { School Buildg }\end{array}$ & 2003 & $\begin{array}{c}\text { No } \\
\text { Investigation }\end{array}$ & None \\
\hline 41. & $\begin{array}{l}\text { \#10 Elas St, } \\
\text { Lagos State }\end{array}$ & $\begin{array}{l}\text { 2-Floor } \\
\text { Residential } \\
\text { Buildg }\end{array}$ & 2004 & $\begin{array}{l}\text { Dilapidated } \\
\text { Structure }\end{array}$ & None \\
\hline 42. & $\begin{array}{l}\text { 22, Makinde } \\
\text { Street, } \\
\text { Ebute-Metta, } \\
\text { Lagos State }\end{array}$ & $\begin{array}{c}\text { Three- Floor } \\
\text { Building }\end{array}$ & 2004 & $\begin{array}{c}\text { No } \\
\text { Investigation }\end{array}$ & None \\
\hline 43. & $\begin{array}{l}\text { 11, Solala } \\
\text { Street, Agege, } \\
\text { Lagos State }\end{array}$ & $\begin{array}{l}\text { 2-Floors } \\
\text { Building }\end{array}$ & 2004 & $\begin{array}{l}\text { No Concluded } \\
\text { Investigation }\end{array}$ & None \\
\hline 44. & $\begin{array}{l}\text { 40, Market } \\
\text { Street, Agege, } \\
\text { Lagos State }\end{array}$ & $\begin{array}{l}\text { 2-Floors } \\
\text { Commercial } \\
\text { Building }\end{array}$ & March 2005 & $\begin{array}{l}\text { No Concluded } \\
\text { Investigation }\end{array}$ & None \\
\hline 45. & $\begin{array}{l}\text { Ibile Holding, } \\
\text { Ikeja, Lagos } \\
\text { State }\end{array}$ & $\begin{array}{l}\text { 2-Floors } \\
\text { Framed } \\
\text { Commercial } \\
\text { Building }\end{array}$ & April 2005 & $\begin{array}{l}\text { No Concluded } \\
\text { Investigation }\end{array}$ & None \\
\hline 46. & $\begin{array}{l}\text { Port Harcourt, } \\
\text { Rivers State }\end{array}$ & $\begin{array}{l}\text { 2-Floors } \\
\text { Commercial } \\
\text { Building }\end{array}$ & June 2005 & $\begin{array}{l}\text { No Concluded } \\
\text { Investigation }\end{array}$ & None \\
\hline 47. & $\begin{array}{c}\text { 6, Princess } \\
\text { Street, Lagos } \\
\text { State }\end{array}$ & $\begin{array}{l}\text { 2-Floors } \\
\text { Commercial } \\
\text { Building }\end{array}$ & July 2005 & $\begin{array}{l}\text { No Concluded } \\
\text { Investigation }\end{array}$ & 1 \\
\hline 48. & $\begin{array}{l}\text { Mushin, Lagos } \\
\text { State }\end{array}$ & $\begin{array}{c}\text { 4-Floors } \\
\text { Residential/ } \\
\text { Commercial } \\
\text { Building }\end{array}$ & Jan. 2005 & $\begin{array}{l}\text { No Concluded } \\
\text { Investigation }\end{array}$ & 1 \\
\hline 49. & $\begin{array}{c}\text { 53, Cemetery } \\
\text { Road, Amukoko } \\
\text { Lagos State }\end{array}$ & $\begin{array}{c}\text { 4-Floors } \\
\text { Residential/ } \\
\text { Commercial } \\
\text { Building }\end{array}$ & Jan. 2006 & $\begin{array}{l}\text { No Concluded } \\
\text { Investigation }\end{array}$ & 7 \\
\hline 50. & $\begin{array}{c}\text { Ikpoba Okha, } \\
\text { Local Govt. Edo } \\
\text { State }\end{array}$ & $\begin{array}{l}\text { 4-Floors } \\
\text { School } \\
\text { Building }\end{array}$ & April 2006 & $\begin{array}{l}\text { No Concluded } \\
\text { Investigation }\end{array}$ & 2 \\
\hline 51. & Abuja, FCT & $\begin{array}{l}\text { 3-Flrs } \\
\text { Building } \\
\text { Housing } \\
\text { Offices and } \\
\text { Churches }\end{array}$ & June 2006 & $\begin{array}{l}\text { No Concluded } \\
\text { Investigation }\end{array}$ & None \\
\hline 52. & $\begin{array}{l}\text { Port Harcourt, } \\
\text { Rivers State }\end{array}$ & $\begin{array}{c}\text { Three-Storey } \\
\text { Residential } \\
\text { Building }\end{array}$ & 2007 & $\begin{array}{l}\text { No Concluded } \\
\text { Investigation }\end{array}$ & 3 \\
\hline 53. & $\begin{array}{l}\text { Ebute-Meta, } \\
\text { Lagos State }\end{array}$ & $\begin{array}{l}\text { Residential/Con } \\
\text { Building }\end{array}$ & 2007 & $\begin{array}{l}\text { Structural } \\
\text { failure }\end{array}$ & None \\
\hline
\end{tabular}

Continued on next page 


\begin{tabular}{|c|c|c|c|c|c|}
\hline \multicolumn{6}{|c|}{ Table 1 continued } \\
\hline 54. & $\begin{array}{c}\text { Kano City, Kano } \\
\text { State }\end{array}$ & $\begin{array}{l}\text { Commercial } \\
\text { Building }\end{array}$ & 2007 & $\begin{array}{l}\text { No concluded } \\
\text { investigation }\end{array}$ & Unspecified \\
\hline 55. & $\begin{array}{c}\text { Abakaliki, } \\
\text { Ebonyi State }\end{array}$ & $\begin{array}{c}\text { 2-Storey } \\
\text { Building } \\
\text { Under } \\
\text { Construction }\end{array}$ & 2011 & $\begin{array}{l}\text { No Concluded } \\
\text { Investigation }\end{array}$ & 3 \\
\hline 56. & $\begin{array}{c}\text { Ikotun, Lagos } \\
\text { State }\end{array}$ & $\begin{array}{c}\text { Synagogue } \\
\text { Church } \\
\text { Building }\end{array}$ & Sept. 2014 & $\begin{array}{l}\text { Excessive } \\
\text { Loading }\end{array}$ & 110 \\
\hline
\end{tabular}

SOURCE: Author Field study, 2021

\begin{tabular}{|c|c|c|c|c|c|}
\hline S/N & Location & Type & Date & Suspected Causes & $\begin{array}{l}\text { Life } \\
\text { lost }\end{array}$ \\
\hline 1. & Isiala Mbano, Imo State & High Court & 1986 & Collapsed ceiling & $\begin{array}{l}\text { Judge } \\
\text { escaped }\end{array}$ \\
\hline 2. & $\begin{array}{l}\text { Imo State University } \\
\text { Owerri, Imo State }\end{array}$ & $\begin{array}{l}\text { Lecture hall } \\
\text { under } \\
\text { construction }\end{array}$ & $\begin{array}{c}\text { Oct } \\
2013\end{array}$ & Excessive loading & None \\
\hline 3. & $\begin{array}{l}\text { Work's layout Owerri, } \\
\text { Imo State }\end{array}$ & $\begin{array}{l}\text { Commercial } \\
\text { building under } \\
\text { contribution }\end{array}$ & $\begin{array}{l}\text { Feb. } \\
2012\end{array}$ & $\begin{array}{l}\text { Excessive loading and use } \\
\text { of substandard materials }\end{array}$ & None \\
\hline 4. & $\begin{array}{c}\text { Egbu road, Owerri, Imo } \\
\text { State }\end{array}$ & $\begin{array}{l}\text { Imo firm } \\
\text { academy just } \\
\text { completed }\end{array}$ & $\begin{array}{l}\text { March } \\
2014\end{array}$ & No investigation & None \\
\hline 5. & Orji, Imo State & $\begin{array}{l}\text { 3-storey hotel } \\
\text { building/un- } \\
\text { der } \\
\text { construction }\end{array}$ & $\begin{array}{l}\text { April } \\
2015\end{array}$ & Error in design & None \\
\hline 6. & $\begin{array}{l}\text { Uzi primary school } \\
\text { Owerri Imo State }\end{array}$ & $\begin{array}{l}\text { School } \\
\text { building }\end{array}$ & $\begin{array}{l}\text { Oct. } \\
2014\end{array}$ & Dilapidated structure & None \\
\hline 7. & $\begin{array}{l}\text { Privately owned located } \\
\text { at Umaru Musa Yar'Adua } \\
\text { Drive, Owerri, Imo State }\end{array}$ & $\begin{array}{l}\text { 8-Storey } \\
\text { building under } \\
\text { construction }\end{array}$ & $\begin{array}{l}30^{t h} \\
\text { April, } \\
2020\end{array}$ & $\begin{array}{l}\text { Disregard to } \\
\text { specifications and other } \\
\text { minimum standards of } \\
\text { construction }\end{array}$ & $\begin{array}{l}\text { Unspec- } \\
\text { ified } \\
\text { but } \\
\text { many }\end{array}$ \\
\hline
\end{tabular}

SOURCE: Author Field study, 2021

\section{RESEARCH METHODOLOGY}

Owerri Municipal is a Local Government Area of Imo State, Nigeria. Its headquarters is in the city of Owerri. Owerri Municipal Council, formerly the headquarters of old Owerri Local Government Area (comprising present day Owerri Municipal, Owerri North, Owerri West and Ngor-Okpala L.G. As) became a municipal council on 15th December, 1996. It's the capital city of Imo State, the eastern heartland, and as such the landlord to most state and federal government ministries, departments and agencies. From the official records of the 2005 national census, the population was about 127,000 people. 
Many business and investment opportunities exist in the municipal council because of the vantage position, the council occupies as the seat of Government and therefore the epicenter of all economic, social, religious activities in the state. In the past Owerri had barely as low as $5 \%$ record of building collapse but in the recent years the occurrence of building collapse in the area has risen and its implication on human lives and properties have given rise to many questions.

\section{POPULATION AND SAMPLING TECHNIQUE}

This study analyzed the views of construction professionals on the causes and remedies of building collapse in Owerri Municipal Council areas (Population). To achieve this goal, a systematic random sampling technique was utilized in the distribution of questionnaire to the concerned sampled construction professionals. The construction professionals were selected from the list provided through membership list solicited from the secretariat of their various State Chapter professional bodies. The professional bodies are the Nigerian Institute of Building (NIOB), Nigerian Institute of Architects (NIA), Nigerian Society of Engineers (NSE) and Niogerian Institute of Quantity Surveyors (NIQS) respectively.

\section{DATA COLLECTION TECHNIQUES}

Primary data were elicited using a well-structured questionnaire in this study whereas the secondary data sources for this study were journals, published articles, and textbooks.

The research involved the use of well-structured four-point Likert Scale questionnaire to solicit information on the perception of construction professionals on the factors causing building collapse and its remedies in the Nigerian construction industry. The factors identified from literature were categorized in tabular format for easy understanding by sampled respondents. The relative importance index method (RII) was used to compare the views of construction professionals within Owerri Municipal Council. This calculation puts the factors in rank order and indicates how much the top ranked is more important than the next and so on. The RII was computed as: Relative importance index (RII) $=\Sigma \mathrm{w} /(\mathrm{Ax} \mathrm{N})---,(0 \leq$ index $\leq 1)$ Where: $\mathrm{w}=$ weighting given to each factor by the respondents and ranges from 1 to 4 where 1 is not significant and 4 is extremely significant, $\mathrm{SA}=$ highest weight (i.e., 4 in this case), and $\mathrm{N}=$ total number of respondents (i.e., in this case 193)

\subsection{SAMPLE SIZE}

The sample size that represents the targeted unknown population was determined from following the equation formula used below.

$$
n=\left(z^{2} p q\right) / d^{2}
$$


Where; $\mathrm{n}=$ the targeted/desired sample size; $\mathrm{z}=$ known as the critical value; $\mathrm{p}=$ the proportion in the target population estimated to have particular character (normally between the range of 0.1-0.5); $\mathrm{q}=1.0-\mathrm{p} ; \mathrm{d}=$ deviation; $\mathrm{P}=$ the proportion in the target population estimated to have particular characteristic (normal between the range of $0.1-0.5) ; \mathrm{q}=1.0-\mathrm{p} ; \mathrm{d}=$ degree of accuracy corresponding to the confidence level $\&$ $\mathrm{Z}$ selected.

Sample size calculation depends on the percentage confidence level and whether it's a known or unknown population.

\section{Confident level and its corresponding $\mathrm{z}$ and $\mathrm{d}$ value.}

1. A $90 \%$ level of confidence has $\alpha=0.10$ and critical value of $\mathrm{z}_{\alpha / 2}=1.64$.

2. A $95 \%$ level of confidence has $\alpha=0.05$ and critical value of $z_{\alpha / 2}=1.96$.

3. A $99 \%$ level of confidence has $\alpha=0.01$ and critical value of $z_{\alpha / 2}=2.58$.

4. A $99.5 \%$ level of confidence has $\alpha=0.005$ and critical value of $\mathrm{z}_{\alpha / 2}=2.81$.

For the purpose of this study, a confidence level of $95 \%$ was adopted owing to the fact that the questionnaire was geared towards assessing the views of construction professionals on causes and remedies of building collapse in the Nigerian construction industry. Consequently, the sample size is determined as thus,

$\mathrm{z}=1.96, \mathrm{~d}=0.05$ where $\mathrm{p}=0.1, \mathrm{q}=0.9 ; \mathrm{N}=\left(1.96^{2} \mathrm{X} 0.2 \times 0.8\right) /(0.05)^{2}=246$

It therefore means that a total of two hundred and forty-six (246) respondents will be sampled in the area using random sampling technique.

\section{TECHNIQUES FOR DATA ANALYSIS}

The data obtained were analyzed using descriptive statistics and use of tables and chart. The descriptive statistics was used to evaluate the relative ranking of those factors that causes collapse of building and its remedy. The results are transformed to relative importance indices based on the likert Scale, to determine the relative ranking of the factors, these scores were then transformed to Relative Importance Index (RII).

$$
R I I=\frac{\sum w}{A N}=\frac{4 n_{4}+3 n_{3}+2 n_{2}+1 n_{1}}{4 N}
$$

Where $w$ is the weighting given to each factor by the respondent, ranging from 1 to 4 , $(n 11=$ number of respondents for option designated as $1, n 2=$ number of respondents for option designated as $2, n 3=$ number of respondents for option designated as $3, n 4$ ), $\mathrm{S} A$ the highest weight (i.e., 4 in the study) and $N$ is the total number of samples. The relative importance index ranges from 0 to 1 . Thus, the questions are in a standardized format and sequence. 


\section{ANALYSIS AND DISCUSSION OF FINDINGS}

The total of 250 questionnaires were distributed to sampled respondents and 193 returned with complete and valid responses whereas 57 of the questionnaires were invalid.

\section{Respondent Profile}

We have 188 respondents to be male, and 5 to be female. The chart below shows that from the respondent profile we have the number of various construction professionals who responded to the questionnaire thus, 50 respondents are Builders, 60 are Engineers, Quantity Surveyors are 40, Architect are 43 to make it a total of 193 respondents.

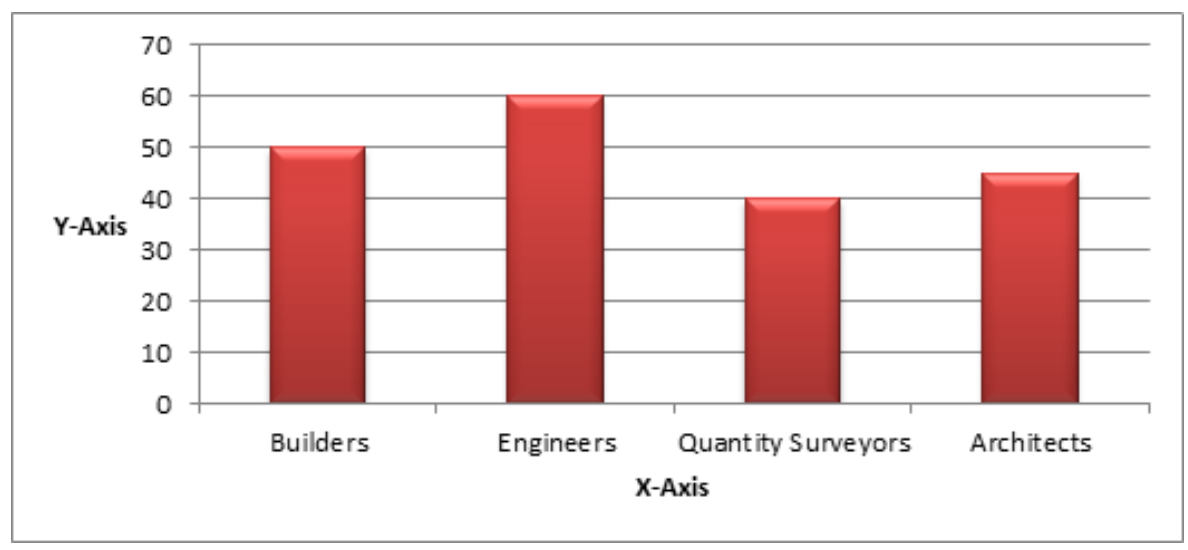

Figure 1 Number of various construction professionalsthat responded to the questionnaire

From the respondent profile we have106 respondents who have been in the construction for 10 years and 77 respondents who have been in the construction for 20 years and 10 respondents for 30 years.

Table 3 Causes of Building Collapse in Owerri, Imo State

\begin{tabular}{llllllllll}
\hline Quantity & SD 1 & D 2 & A 3 & SA 4 & $\sum \mathbf{f}$ & $\sum \mathbf{f x}$ & Mean & RII & Ranking \\
Soil Type & - & - & 66 & 127 & 193 & 706 & 3.66 & 0.91 & $3^{\text {rd }}$ \\
Poor building design and planning & 16 & 20 & 60 & 97 & 193 & 624 & 3.23 & 0.81 & $5^{\text {th }}$ \\
Use of low-quality building materials & - & 7 & 36 & 150 & 193 & 722 & 3.74 & 0.94 & $2^{\text {nd }}$ \\
Use of incompetent craftsmen & 20 & 10 & 57 & 106 & 193 & 635 & 3.29 & 0.82 & $4^{\text {th }}$ \\
Weak supervisor & 115 & 40 & 22 & 16 & 193 & 325 & 1.68 & 0.42 & $7^{\text {th }}$ \\
Use of non-professional & 60 & 130 & 22 & - & 193 & 329 & 1.70 & 0.43 & $6^{\text {th }}$ \\
Corruption & - & - & 26 & 167 & 193 & 746 & 3.87 & 0.97 & $1^{\text {st }}$ \\
\hline
\end{tabular}

Results obtained from Table 3 indicates that Corruption is ranked $1^{\text {st }}$ position on the causes of building collapse with RII of 0.97 and closely followed is, use of lowquality building materials with RII of 0.94 ranked $2^{\text {nd }}$ and soil type, which ranked $3^{\text {rd }}$ has RII of 0.91 . however, the result obtained from Table 3 indicates that, weak supervision with RII of 0.42 ranked $7^{\text {th }}$ and the list amongst identified causes of build- 
ing collapse. The results obtained from this Table and section may imply that once corruption is tackled the rest of the factors can be controlled easily.

\begin{tabular}{|c|c|c|c|c|c|c|c|c|c|}
\hline Quantity & SD 1 & D 2 & A 3 & SA 4 & $\sum \mathbf{f}$ & $\sum \mathbf{f} \mathbf{x}$ & Mean & RII & Ranking \\
\hline $\begin{array}{l}\text { Destroys lives } \\
\text { and properties }\end{array}$ & - & 7 & 26 & 160 & 193 & 732 & 3.79 & 0.95 & $1^{s t}$ \\
\hline $\begin{array}{c}\text { Affects } \\
\text { socio-economy }\end{array}$ & - & 56 & 37 & 100 & 193 & 623 & 3.23 & 0.81 & $4^{t h}$ \\
\hline $\begin{array}{c}\text { Keeps } \\
\text { occupants and } \\
\text { house owners } \\
\text { out of their } \\
\text { house }\end{array}$ & - & - & 42 & 151 & 193 & 730 & 3.78 & 0.95 & $1^{s t}$ \\
\hline $\begin{array}{c}\text { Causes } \\
\text { additional cost } \\
\text { on a particular } \\
\text { project }\end{array}$ & - & - & 97 & 96 & 193 & 675 & 3.50 & 0.88 & $3^{\text {rd }}$ \\
\hline
\end{tabular}

In similar development, results obtained from Table 4 reveal that amongst the problems associated to building collapse identified, destroys life and properties as well as keeps occupants and house owners out of this house, tied in the $1^{\text {st }}$ position with RII values of 0.95 respectively. Whereas, causes additional cost on a particular project, ranked $2^{\text {nd }}$ position with RII $(0.88)$, and affects socio-economy ranked $4^{\text {th }}$ and last position with RII (0.81). This shows that the major problems associated to building collapse is 'destroys life and properties as well as rendering the occupants of the building and the owner homeless or houseless. The results obtained in Table 4 corroborated the view of literature that the implications from the menace of building collapse wreaks havoc on the people and the environment.

Table 5 Remedies Associated to Building Collapse in Owerri, Imo State

\begin{tabular}{|c|c|c|c|c|c|c|c|c|c|}
\hline Quantity & $\begin{array}{c}\text { SD } \\
1\end{array}$ & $\begin{array}{l}\text { D } \\
2\end{array}$ & $\begin{array}{l}\text { A } \\
3\end{array}$ & $\begin{array}{c}\text { SA } \\
4\end{array}$ & $\sum \mathbf{f}$ & $\sum \mathbf{f x}$ & Mear & RII & $\begin{array}{c}\text { Rank- } \\
\text { ing }\end{array}$ \\
\hline Avoid the use of quacks. & - & - & 20 & 173 & 193 & 752 & 3.90 & 0.98 & $2^{\text {nd }}$ \\
\hline Adherence to designed reinforcement. & - & - & 2 & 191 & 193 & 770 & 3.99 & 0.99 & $1^{\text {st }}$ \\
\hline Use of high-quality materials. & 5 & - & 11 & 177 & 193 & 746 & 3.87 & 0.97 & $4^{t h}$ \\
\hline Constant's supervision. & 21 & 7 & 64 & 101 & 193 & 631 & 3.27 & 0.82 & $5^{t h}$ \\
\hline $\begin{array}{l}\text { Soil test must be carried out to determine } \\
\text { the soil bearing capacity. }\end{array}$ & - & - & 19 & 174 & 193 & 753 & 3.90 & 0.98 & $2^{n d}$ \\
\hline
\end{tabular}

Results obtained in Table 5 shows the response from the respondents on the (remedies) ways to prevent the collapse of building in Owerri, Imo State Nigeria. We have, adherence to designed reinforcement details which have theRIIof 0.99 ranking $1^{\text {st }}$, avoid the use of non-professional (quack) and testing of soil to 0.97 ranking $4^{\text {th }}$ while the constant supervision has the RIIof 0.82 Ranking $5^{\text {th }}$ in the table. This shows that adherence to designed reinforcement details and use of professional in 
every stage of the constructions project will go a long way in controlling causes of collapse of a building.

Table 6 Suggested ways for Effective Regulatory Controls for Building Compliance to National Building Code to Mitigate Building Collapse.

\begin{tabular}{|c|c|c|c|c|c|c|c|c|c|}
\hline Quantity & $\begin{array}{c}\text { SD } \\
1\end{array}$ & $\begin{array}{l}D \\
2\end{array}$ & A 3 & SA 4 & $\sum \mathbf{f}$ & $\sum \mathbf{f x}$ & mean & RII & Rankin \\
\hline $\begin{array}{l}\text { Building projects must } \\
\text { bear the seals of } \\
\text { construction } \\
\text { professionals involved. }\end{array}$ & - & - & 20 & 173 & 193 & 752 & 3.90 & 0.98 & $1^{\text {st }}$ \\
\hline $\begin{array}{l}\text { Government agencies } \\
\text { for approval of building } \\
\text { plan must not collect } \\
\text { bribe from developers } \\
\text { or contractors. }\end{array}$ & - & - & 60 & 133 & 193 & 712 & 3.69 & 0.92 & $5^{t h}$ \\
\hline $\begin{array}{l}\text { Every building design } \\
\text { must be approved by } \\
\text { qualified professionals } \\
\text { before commencement } \\
\text { of works. }\end{array}$ & - & - & 31 & 162 & 193 & 741 & 3.84 & 0.96 & $4^{t h}$ \\
\hline $\begin{array}{l}\text { Any building project } \\
\text { that doesn't meet the } \\
\text { requirement of the } \\
\text { national building code } \\
\text { must not be built. }\end{array}$ & - & - & 13 & 180 & 193 & 759 & 3.93 & 0.98 & $1^{s t}$ \\
\hline $\begin{array}{l}\text { Government must } \\
\text { award contract direct } \\
\text { to certified } \\
\text { professionals and not } \\
\text { political counterpart. }\end{array}$ & - & - & 22 & 171 & 193 & 750 & 3.89 & 0.97 & $3^{r d}$ \\
\hline
\end{tabular}

The results shown in Table 6 indicates that all 'building project must bear seals from construction professionals before commencement of works' with RII 0.98 ranked $1^{\text {st }}$ position and tied with 'any building that doesn't meet the requirement of national building code must not be built' with RII of 0.98 amongst the five identified suggested ways of ensuring compliance to statutory regulations as specified in the national building code. However, 'government should award contracts to satisfied professionals and not to political counterparts' has RII of 0.97 and ranked $2^{n d}$, whereas 'every building must be approved by professional from the design team' ranked $4^{t h}$ position with RII of 0.96. Finally, 'government agencies for approval of building plan must not collect bribe from the developers or contractors' ranked $5^{\text {th }}$ and last position with RII of 0.92 . to this end, the results obtained implies that for building projects to stand the test of time it must have stamp and seals of the professionals involved in the project. If this measure is complied with, it is believed that it will further add towards the control of the incidences of building collapse across Nigeria. 


\section{SUMMARY OF FINDINGS}

The information gathered and analyzed from the questionnaire had undoubtedly underscored the views of the construction professionals on the causes and remedies of building collapse in Nigeria. Results contained in Table 3 indicate that 'Corruption' has been implicated to be the major causes of building collapse followed by 'use of low-quality materials. This study believes strongly that when corruption is tackled by the government and the professional bodies including other critical stakeholders all other factors identified will be eradicated.

In similar development, results obtained from Table 4 reveals that 'destruction of lives and properties' which tied with 'keeps occupants and house owners' emerged $1^{\text {st }}$ position. This implies that when buildings collapse it either kills the occupants and or destroys their properties, and the occurrence further renders them homeless, emotionally wrecked and financially destabilized. In Table 5 , 'adherence to designed reinforcement details' had RII of 0.99 and ranked $1^{\text {st }}$ position whereas 'constant supervision ranked $5^{\text {th }}$ position amongst the remedies associated to building collapse. This implies that it is only an incorrupt professional that can adhere to the designed details and carry out soil testing and make use of high-quality material to achieve a high degree of construction work that will last the text of time.

In the same light, results obtained in Table 6 indicates that for effective regulatory control of compliance to building construction practice as stipulated in the national building code, completed projects or those under construction must bear seals of construction professionals who supervised or are supervising the works in progress. Furthermore, when all these articulated points are adhered to, the incidences of building collapse would be drastically reduced to its barest minimum if not totally eliminated.

\section{CONCLUSION AND RECOMMENDATIONS}

The problem of building collapse in Nigeria will continue reverberate in this country unless all critical stakeholders work together with a sincerity of purpose to stem the tide. It has been stated that the building collapse phenomena is largely traceable to unethical practices of some professional stakeholders and developers, including government officials. It must be mentioned that existing regulations and codes are enough to deal with the situation, but these are often adhered to in breach than in observance. The study believes that some government officials contribute to this mess as they collect gratifications and award contracts to incompetent contractors and even issue approvals without actually scrutinizing the projects. Such compromises have caused enormous cost and liabilities to the society who often becomes victims of the collapsed building. Additionally, it is further believed that when buildings collapses, clients do not get value for their investments.

The construction industry will be better able to continue to provide the quality services on which society increasingly depends. Professionals should endeavor to 
attend conferences /workshops and seminars both local and overseas so as not to put themselves out of rhythm. It further recommends the formation of a Federal Building Safety Corps to address all problems of construction in buildings. Contractors are charged to use good quality materials as must have been specified in the course of construction. At the same time, Government and developers should also deploy the services of construction professionals to every project as stipulated by existing statutes as this will greatly avert the incidences of building collapse in Nigeria.

\section{REFERENCES}

Akinpelu, A. O., Iyaniwura, J. O., \& Ajagbe, B. O. (2001). The reliability of Berger's table in estimating 1-RM and 10-RM of the elbow flexor muscles in normal young adults. In South African Journal of Physiotherapy (Vol. 57, pp. 11-15). Nigeria Institute Of Building: AOSIS. Retrieved from https://dx.doi.org/10.4102/sajp.v57i2.499 10.4102/sajp .v57i2.499

Anosike, N. M., \& Inyang-Udoh, U. (2015). Investigating Construction Malpractices In The Nigerian Construction Industry. Nicmar Journal of Construction Management, 1-15.

Ayedun, C. A., Durodola, O. D., \& Akinjare, O. A. (2012). An Empirical Ascertainment Of The Causes Of Building Failure And Collapse In Nigeria”. Mediterranean Journal of Social Sciences, 3(1), 313-324.

Ayininuola, G. M., \& Olalusi, O. O. (2005). ASSESSMENT OF BUILDING FAILURES IN NIGERIA: LAGOS AND IBADAN CASE STUDY. African Journal of Science and Technology, 5(1), 73-78. Retrieved from https://dx.doi.org/10.4314/ajst.v5i1.15321 10.4314/ajst.v5i1 .15321

Ayuba, P., Olagunju, R. E., \& Akande, O. K. (2012). Failure And Collapse Of Buildings In Nigeria: The Role Of Professionals And Other Participants In The Building Industry". Interdisciplinary Journal Of Contemporary Research In Business, 4(6), 1267-1272.

Dimuna, K. O. (2010). Incessant Incidents Of Building Collapse In Nigeria: A Challenge To Stakeholders". Global Journal of Researches In Engineering, 10, 75-80.

Ede, A. N. (2000). Building Collapse In Nigeria: The Trend Of Casualties In The Last Decade. International Journal Of Civil And Environmental Engineering, Ijcee-Ijens, 10(06), 32-42.

Enenmoh, C. R., \& Anosike, N. M. (2017). Client's Obligational Negligence In Nigerian Construction Industry. International Journal For Research \& Development In Technology (Ijrdt), 4(8), 1-9.

Fakere, A. A., Fadairo, G., \& Fakere, R. A. (2012). Assessment Of Building Collapse In Nigeria: A Case Of Naval Building. International Journal Of Engineering And Technology, 2(4), 584-590.

Fredrick, M., \& James, A. (1989). Building Engineering And Systems Design. (Vol. 2). New York: Van Nostrand Reinhold.

Hall, G. T. (1984). Revision Notes On Building Maintenance And Adaptation.

Ibrahim, R. B. (2013). Monumental Effects Of Building Collapse In Nigeria Cities: The Case Of Lagos Island. Research Journal Of Engineering Innovation, 1(2), 26-31.

Nbrri. (2020). Nigerian Building \& Road Research Institute:0 Nbrri Report No. 51 "Technical Report On The Collapsed 8-Storey Building Under Construction At Umaru Musa Yar'adua Drive.

Olagunju, R. E., Aremu, S. C., \& Ogundele, J. (2013). Incessant Collapse Of Building In Nigeria: An Architect's View". Civil And Environmental Research, 3(4), 2225-0514. 
Ukpata, J. O., \& Sunday 15th October. (2006). Structural Design As A Panacea For Building Failures In Nigeria", A Paper Presented On The Occasion To Mark The Vocational Service Month Of Hilltop. 